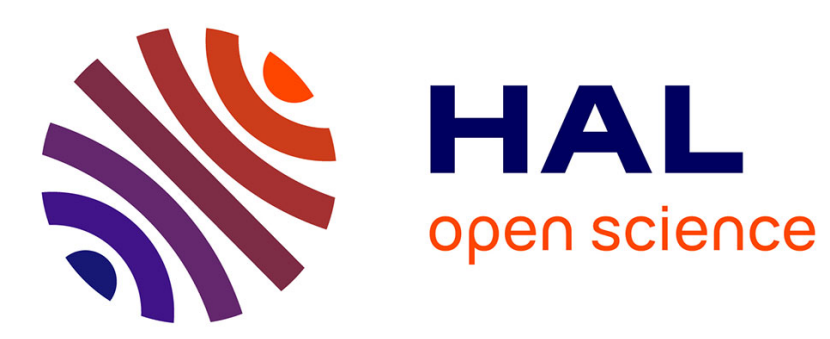

\title{
LES of the flow around several cuboids in a row
}

David Uystepruyst, Siniša Krajnovic

\section{To cite this version:}

David Uystepruyst, Siniša Krajnovic. LES of the flow around several cuboids in a row. International Journal of Heat and Fluid Flow, 2013, 44, pp.414 - 424. 10.1016/j.ijheatfluidflow.2013.07.011 . hal01086410

\section{HAL Id: hal-01086410 https://hal.science/hal-01086410}

Submitted on 24 Nov 2014

HAL is a multi-disciplinary open access archive for the deposit and dissemination of scientific research documents, whether they are published or not. The documents may come from teaching and research institutions in France or abroad, or from public or private research centers.
L'archive ouverte pluridisciplinaire $\mathbf{H A L}$, est destinée au dépôt et à la diffusion de documents scientifiques de niveau recherche, publiés ou non, émanant des établissements d'enseignement et de recherche français ou étrangers, des laboratoires publics ou privés. 


\title{
LES of the flow around several cuboids in a row
}

\author{
David Uystepruyst ${ }^{\mathrm{a}}$, Siniša Krajnovića \\ ${ }^{a}$ Division of Fluid Dynamics, Department of Applied Mechanics, Chalmers University of \\ Technology, SE-41296 Gothenburg, Sweden.
}

\section{Abstract}

This paper presents Large Eddy Simulations (LES) of flow around a fourvehicle platoon when one of the platoon members was forced to undergo in-line oscillations. The LES were made at the Reynolds number of $10^{5}$ based on the height of the vehicles. Combinations of two different frequencies corresponding to non-dimensional frequencies at the Strouhal numbers $S t_{1}=0.025$ and $S t_{2}=0.013$ and two oscillation amplitudes were used in this study. The methodology was validated by comparisons with data from previous experimental investigations. In order to highlight the dynamic effects, comparisons were made with steady results on a single vehicle and on a four-vehicle platoon. Large differences were found in the flow structures between quasi-steady and dynamic results. Furthermore, the behaviour of the drag coefficient of the upstream neighbor of the oscillating model was 
investigated.

Keywords: Unsteady vehicle aerodynamics, LES, Grid deformation, Platooning, Vehicle, Hysteresis

\section{Introduction}

A platoon consists of a group of close-following vehicles equipped with autopilot, sensors, computers and communications hardware to allow high speed travel at small inter-vehicle spacings. The first advantage is to increase the highway throughput thanks to the inter-vehicle spacing reduction.

The minimal inter-vehicle spacing yields an increase of the base pressure and a decrease of the forebody pressure of the platoon members. This leads to a drag reduction and, hence, savings in fuel consumption which constitutes the second main advantage.

However, it was shown $[9,15]$ that, in certain circumstances, especially with a tandem configuration, the improvement of aerodynamic performances is negligible. For close spacing, there can even be significant drag penalties [9]. Fortunately, as soon as the number of platoon members is at least three, all 
studies demonstrated an efficient global drag reduction [6, 13, 14]. Moreover, Tsuei and Savaş [14] showed that the averaged drag of a vehicle platoon decreases when the platoon size increases.

Another important point for the platoon performances is the shape of vehicles. Thus, a hatchback vehicle, like the Golf-class car, experiences a lower drag reduction than a squareback vehicle, like a minivan or compact car, in a platoon configuration $[14,11]$.

Tandem or platoon configurations are beside vehicle applications relevant for applications with other bluff bodies. This is why some studies were realized in order to understand the flow behaviour around bluff bodies, like disks or cylinders $[2,17]$ or cubes $[7,10]$. These studies were, in particular, dedicated to the periodic vortex shedding occurring in the gap between bluff bodies.

Some experimental aerodynamic studies are dedicated to the inter-vehicle spacing effect $[9,15,6,13,14,16,1]$ on a platoon or tandem. The majority 
of these studies is limited to steady measurements. However, it is a real challenge to maintain a constant inter-vehicle spacing however, and the platoon members inevitably oscillate. These dynamic oscillations have undeniable effects on the aerodynamic performances of the platoon. The explanation for the lack of unsteady studies is simply that they are much more difficult to perform, experimentally and numerically.

Transient wind tunnel experiments with vehicle platoons were carried out by Tsuei and Savaş [14]. They made comparisons of quasi-steady and unsteady configurations by moving the third member of a four-vehicle platoon. The position of the moving member was set by a sinusoidal function. Different aspects, such as the displacement amplitude, the frequency of oscillations and the shape of the model were studied. It was shown that the oscillation of the third model had a strong influence on the drag force of the neighbor members. The upstream neighbor especially experienced a chaotically changing drag. 
The present work aims to analyze the flow involved in a car platoon configuration. The simulation technique of large eddy simulation is used to accurately predict the flows in different scenarios (with one or several models, quasi-steady and dynamic motion) and to give an explanation of the physical phenomena involved. This introductory part of the paper is followed by a brief description of the experimental model that is used in the present numerical study, in section 2.1. This is followed by a description of the numerical set-up in section 2.2. The results of the LES are then provided and analyzed in section 3. The paper is summarized and conclusions are drawn in section 4.

When the oscillating body is moving, the case will be denoted as quasi-steady or dynamic depending on whether the third model is at a constant position or moving continuously, respectively.

\section{Method}

\subsection{Description of the experimental model}

The models consisted of simple sharped-edge rectangular boxes, shown in

figure 1, representing a simplified geometry of a minivan. The dimensions of 


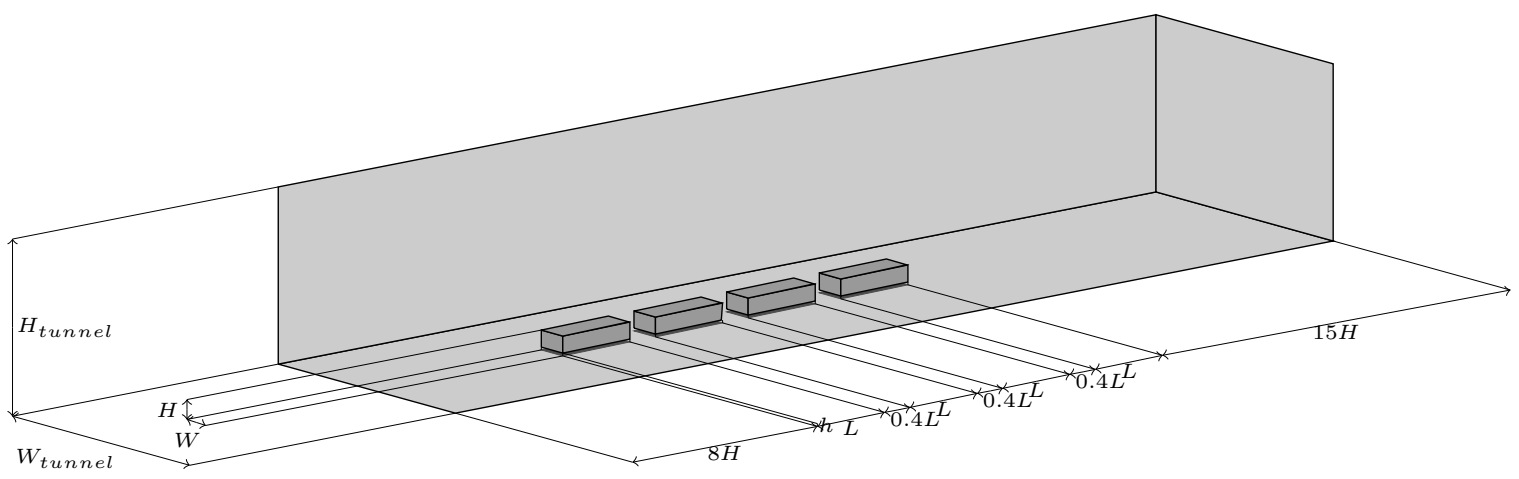

Figure 1: Wind tunnel and location of the four models in the uniform intra-platoon spacing.

\begin{tabular}{llllll}
\hline$L$ & $W$ & $H$ & $h$ & $W_{\text {tunnel }}$ & $H_{\text {tunnel }}$ \\
\hline 0.254 & 0.102 & 0.089 & 0.013 & 0.81 & 0.81 \\
\hline
\end{tabular}

Table 1: Dimensions of the models and the wind-tunnel, in $m$.

the models, as well as the dimensions of the wind tunnel, are reported in table 1 . The model length was $L=0.254 \mathrm{~m}$, which represents a $1 / 20 t h$ model scale. The inter-vehicle spacing was $0.4 L \simeq 0.102 \mathrm{~m}$ and was thus equivalent to $2 \mathrm{~m}$ in full scale. The model height was $0.076 \mathrm{~m}$, giving a Reynolds number of $R e_{H}=10^{5}$ for a wind tunnel velocity of $U_{\infty}=20 \mathrm{m.s} \mathrm{s}^{-1}$. Note that the width and the height of the wind tunnel were the same as in the experimental work of Tsuei and Savaş [14]. 
To demonstrate the effect of platooning, steady measurements of the drag coefficient of each member in the four-vehicle platoon with an uniform intraplatoon spacing were made and compared with the single-vehicle value. Measurements were then made with third model at both quasi-steady positions and dynamic motion. The quasi-steady measurements were carried out for nine positions of the oscillating vehicle: $d=0, \pm 0.019, \pm 0.038, \pm 0.057$ and $\pm 0.076 \mathrm{~m}$. The negative values corresponded to the third body closer to the fourth one, while positive values were for the third body closer to the second one. In this numerical work, position $d$ will be divided by the original intra-platoon spacing, $0.4 L$, for the sake of generality, to give $\delta=d / 0.4 L$. Therefore, the positions $d=0, \pm 0.019, \pm 0.038, \pm 0.057$ and $\pm 0.076 \mathrm{~m}$ are equivalent to the positions $\delta=0, \pm 0.1875, \pm 0.375, \pm 0.56$ and $\pm 0.75 \mathrm{~m}$. For transient simulations, the displacement amplitudes, $d_{a m p}$, were set to 0.038 and $0.076 \mathrm{~m}$ and the velocity amplitudes, $v_{a m p}$, ranged from 0.25 to $1 \mathrm{m.s}^{-1}$. Actually, the highest amplitude, $0.076 \mathrm{~m}$, was really about $0.07 \mathrm{~m}$ in the experimental work of Tsuei and Savaş [14]. However, the number $0.076 \mathrm{~m}$ was still used in the experimental work and will be used in this present paper, for 
convenience. A smooth, periodic motion had been successfully simulated by expressing the oscillating vehicle's position and velocity as sinusoidal functions:

$$
\begin{aligned}
& d(t)=-d_{a m p} \sin (2 \pi f t), \\
& v(t)=\dot{d}(t)=-v_{a m p} \cos (2 \pi f t)=-2 \pi f d_{a m p} \cos (2 \pi f t),
\end{aligned}
$$

where $f$ is the oscillation frequency and $t$ is the time. These positions Effects of platooning were highlighted by analyzing the aerodynamic drag force $F_{D}$ which was scaled to obtain the aerodynamic drag coefficient $C_{d}$ :

$$
C_{d}=\frac{F_{d}}{\frac{1}{2} \rho U_{\infty}^{2} S}
$$

where $\rho$ is the fluid density and $S$ is the frontal area of the models:

$$
S=W(H-h)
$$

where $W$ is the width, $H$ is the total height and $h$ is the ground clearance as defined in table 1.

\subsection{Numerical set-up}

\subsubsection{LES governing equations}

The governing LES equations were the incompressible Navier-Stokes and the continuity equations filtered with the implicit spatial filter of character- 
istic width $\Delta$

$$
\frac{\partial \bar{u}_{i}}{\partial t}+\frac{\partial}{\partial x_{j}}\left(\bar{u}_{i} \bar{u}_{j}\right)=-\frac{1}{\rho} \frac{\partial \bar{p}}{\partial x_{i}}+\nu \frac{\partial^{2} \bar{u}_{i}}{\partial x_{j} \partial x_{j}}-\frac{\partial \tau_{i j}}{\partial x_{j}}
$$

and

$$
\frac{\partial \bar{u}_{i}}{\partial x_{i}}=0
$$

Here, $\bar{u}_{i}$ and $\bar{p}$ are the resolved velocity and pressure, respectively, and the bar over the variables denotes filtering.

The influence of the small scales of the turbulence on the large energy carrying scales in Eq. (4) appears in the SGS stress tensor, $\tau_{i j}=\overline{u_{i} u_{j}}-\bar{u}_{i} \bar{u}_{j}$. The algebraic eddy viscosity model originally proposed by Smagorinsky [12] was used in the present work for its simplicity and low computational cost. The Smagorinsky model represents the anisotropic part of the SGS stress tensor, $\tau_{i j}$, as

$$
\tau_{i j}-\frac{1}{3} \delta_{i j} \tau_{k k}=-2 \nu_{S G S} \bar{S}_{i j}
$$

where $\nu_{S G S}=\left(C_{s} f \Delta\right)^{2}|\bar{S}|$ is the SGS viscosity and

$$
\bar{S}_{i j}=\frac{1}{2}\left(\frac{\partial \bar{u}_{i}}{\partial x_{j}}+\frac{\partial \bar{u}_{j}}{\partial x_{i}}\right)
$$


is the resolved rate-of-strain tensor and $|\bar{S}|=2\left(\bar{S}_{i j} \bar{S}_{i j}\right)^{1 / 2}$. The value of the Smagorinsky constant $C_{s}=0.1$ previously used for similar types of vehicle bluff body flows $[3,8]$ was used in the present work. $f$ in the expression for the SGS viscosity is the van Driest damping function

$$
f=1-\exp \left(\frac{-n^{+}}{25}\right)
$$

where $n^{+}$is the wall normal distance in viscous units. The filter width, $\Delta$, is defined in this work as $\Delta=\left(\Delta_{1} \Delta_{2} \Delta_{3}\right)^{1 / 3}$, where $\Delta_{i}$ are the computational cell sizes in the three coordinate directions.

\subsubsection{Numerical method}

The incompressible equations, (4) and (5), were solved using a commercial finite volume solver, AVL Fire. A collocated grid arrangement was employed. The convective fluxes were approximated by a blend of $95 \%$ linear interpolation of second order accuracy (central differencing scheme) and of $5 \%$ upwind differences of first order accuracy (upwind scheme). The diffusive terms containing viscous plus sub-grid terms were approximated by a central differencing interpolation of second order accuracy. The time marching procedure was done using the implicit second order accurate three-time level 
scheme

$$
\left(\frac{d \phi}{\partial t}\right)_{n+1}=\frac{3 \phi^{n+1}-4 \phi^{n}+\phi^{n-1}}{2 \Delta t}
$$

where indices $n+1$ and $n$ denote the new and the old time iterations, respectively. The SIMPLE algorithm was used to update the pressure and velocity fields to satisfy the continuity equation.

\subsubsection{Numerical details}

The models were placed in a domain as shown in figure 1. A uniform velocity profile of $U_{\infty}=20 \mathrm{~m} . \mathrm{s}^{-1}$ was used at the inlet, which was the same as used in the experimental tests. The boundary layer thickness measured in the experiment was $1.9 \mathrm{~mm}$ at the section entrance and $4.4 \mathrm{~mm}$ at $108 \mathrm{~cm}$ downstream. Here, the inlet condition was set to obtain the same thickness of boundary layer at the front of the first model. The homogeneous Neumann boundary condition was applied at the outlet. The lateral surfaces and the ceiling of the wind tunnel were treated as slip surfaces. Finally, no-slip boundary conditions were used on the surface of the body and the channel floor. 
Numerical accuracy was established by making simulations on two different multi-block hexahedral computational grids. A grid topology was constructed using several O-grids in order to concentrate most of the computational cells close to the surface of the models, see figure 2 .

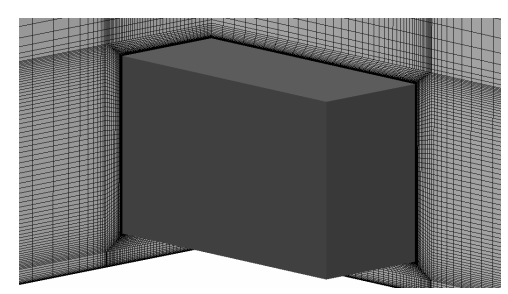

Figure 2: Perspective view of the mesh around a model.

The coarse grids consisted of 1.8 and 5 million cells for the case with a single model and the case with a four-model platoon, respectively, see table 2 . The fine grids consisted of 2.8 and 7.9 million cells.

For both the coarse and the fine grids, the averaged wall normal resolution on the model was $n^{+} \simeq 0.65$. The main differences between coarse and fine grids were the averaged resolution in streamwise and spanwise directions. For the coarse grids, the resolution was $6<\Delta s^{+}<150$ in the streamwise 


\begin{tabular}{l|l|l|l|l}
\hline & \multicolumn{2}{|c|}{ Coarse } & \multicolumn{2}{c}{ Fine } \\
\hline Configuration & single & platoon & single & platoon \\
\hline Num. elements & 1.8 & 5 & 2.8 & 7.9 \\
\hline \multirow{2}{*}{ Resolution } & \multicolumn{3}{|c|}{$6<\Delta l^{+}<45$} & \multicolumn{2}{c}{$6<\Delta l^{+}<33$} \\
& \multicolumn{3}{|c|}{$6<\Delta s^{+}<150$} & \multicolumn{3}{c}{$6<\Delta s^{+}<106$} \\
\hline
\end{tabular}

Table 2: Number of elements, in millions, and spatial resolution for the different meshes.

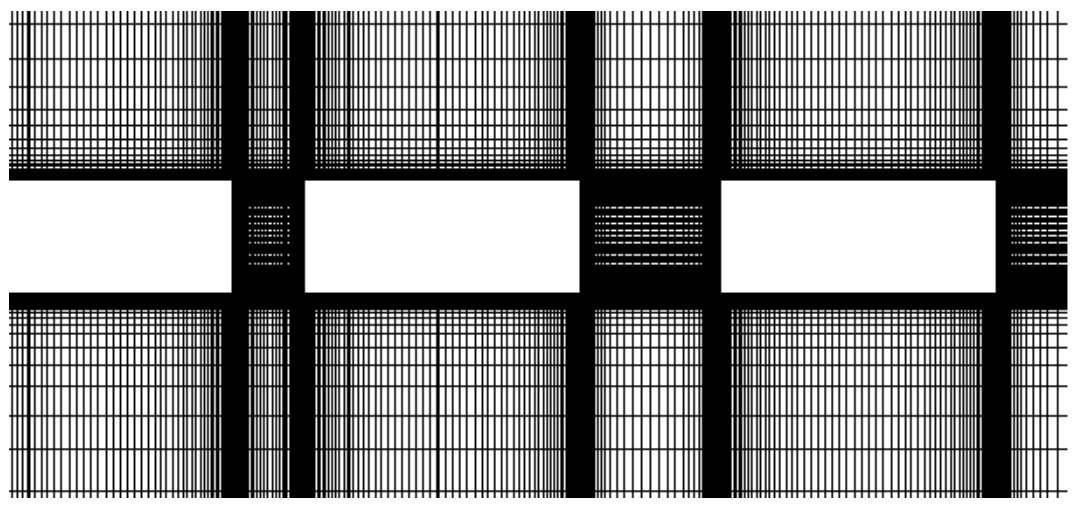

(a) $\delta=0.375$

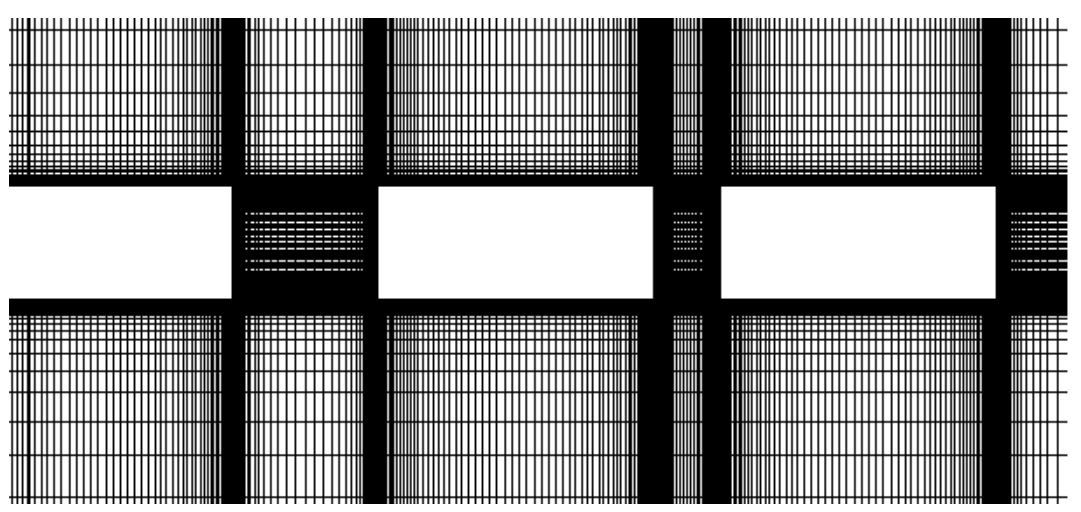

(b) $\delta=-0.375$

Figure 3: Effect of the deformation on the computational grid. 
direction and $6<\Delta l^{+}<45$ in the spanwise direction. The fine grid had $6<\Delta s^{+}<106$ and $6<\Delta l^{+}<33$. The mean values of $\Delta s^{+}$were 80 and 50 in the coarse and the fine grids, respectively. The corresponding mean values of $\Delta l^{+}$were 25 and 20 in the coarse and the fine grids, respectively. Here, $\Delta n^{+}=n<u_{\tau}>_{t} / \nu, \Delta s^{+}=\Delta s<u_{\tau}>_{t} / \nu$ and $\Delta l^{+}=\Delta l<u_{\tau}>_{t} / \nu$, and $\left\langle u_{\tau}\right\rangle_{t}$ is the time-averaged friction velocity. The time step for both the coarse and the fine grids was $\Delta t=2 \times 10^{-4} \mathrm{~s}$, giving a CFL number below one in over $99 \%$ of the cells. The steady-body calculations were run for time $t U_{\infty} / H \simeq 780$. Afterwards, the dynamic calculations were started from the flow resulting from steady-body calculations and run during a time period of $t U_{\infty} / H \simeq 1300$ and $t U_{\infty} / H \simeq 2600$ for the cases with the frequency $f=2 \mathrm{~Hz}$ and $f=1 \mathrm{~Hz}$, respectively. For both cases, the running time corresponded to 10 complete oscillation periods of the third model. After the switch from the steady-body simulation to the dynamic simulation, the LES were run for one complete oscillation period of the third model before the results were monitored. The drag coefficients of the following dynamic model were averaged on the nine last oscillation periods of the third model. 
All the results were filtered with a $8 \mathrm{~Hz}$ low-pass Butterworth filter, i.e. with the same frequency used in the experimental work. This filter was also used to simplify the comparisons with experimental data which were difficult with all the spurious oscillations given by the LES.

The oscillation of the third model along the x-axis in the present LES was obtained by deforming the computational grid in the AVL FIRE software. The effect of the deformation is illustrated in figure 3. The deforming of the grid was done in the two regions defined as the gap between the second and the third models, and between the third and the fourth models, respectively. The cells located in the O-blocks were not altered by the deformations. A similar approach was successfully used by Krajnović et al. [4, 5] for the unsteady RANS simulations of trains passing each other or exiting a tunnel and for the LES simulations of a rotating vehicle.

\section{Results}

To highlight the platooning effect, steady-body LES calculations were performed on a single model and on a four-model platoon. Five positions were 
studied with quasi-steady LES simulations: $d=0, \pm 0.038 \mathrm{~m}$ and $\pm 0.076 \mathrm{~m}$ are equivalent to the positions $\delta=0, \pm 0.375$ and \pm 0.75 . These positions were chosen to perform comparisons with the transient results, experimental and numerical, for which the amplitudes were $\delta_{a m p}=0.375$ and $\delta_{a m p}=0.75$. Dynamic oscillations were performed for different frequencies and different amplitudes. Experimental data presented here come from Tsuei and Savaş $[14]$.

\subsection{Steady and quasi-steady results}

\subsubsection{Single model and uniform intra-platoon spacing}

Table 3 gives the values of the experimental and numerical drag coefficients for the steady-body configurations. The first row represents the values obtained with a single model. The last four rows list the values obtained for each model in the platoon configuration.

As can be seen, relative differences between the experiments and the LES on the single model are 11 and 6 per cent for the coarse and the fine grids, respectively. They are 16 and 11 per cent for the first model of the platoon configuration. The results obtained for the three other models are closer to 


\begin{tabular}{l|l|l|l|l|l|l|l|l}
\hline $\mathrm{N}$ & Member & Experiment & \multicolumn{3}{|c|}{ Coarse } & \multicolumn{3}{c}{ Fine } \\
\hline & & $C_{d}$ & $C_{d}$ & rms & $\epsilon \%$ & $C_{d}$ & rms & $\epsilon \%$ \\
\hline 1 & box & 0.996 & 0.888 & 0.011 & 11 & 0.935 & 0.015 & 6 \\
\hline \multirow{4}{*}{4} & box1 & 0.867 & 0.725 & 0.021 & 16 & 0.773 & 0.02 & 11 \\
& box2 & 0.313 & 0.307 & 0.032 & 2 & 0.287 & 0.04 & 8 \\
& box3 & 0.225 & 0.222 & 0.032 & 1 & 0.216 & 0.036 & 4 \\
& box4 & 0.359 & 0.317 & 0.026 & 12 & 0.342 & 0.03 & 5 \\
\hline
\end{tabular}

Table 3: Drag coefficient values for the steady simulations, for a single model $(\mathrm{N}=1)$ and four-model platoon $(\mathrm{N}=4)$, together with experimental data. $\epsilon$ represents the relative difference between experimental and numerical drag coefficients.

the experiments with, in particular, differences lower than $10 \%$ for the fine grid. As the last three models are located behind the first one, a drafting effect occurs on them. That means that the velocities impinging the three last models are lower than the inlet velocity impinging the first model. The resolution is therefore better for the three last models than for the first one. The results are hence better.

Tsuei and Savaş [14] reported that there was a problem on the force balance measuring the aerodynamic forces of the first model of the platoon. It can also explain the large discrepancy between the experimental data and nu- 
merical results for this model.

The platoon effect is highlighted in figure 4, which shows the drag coefficients of each platoon member normalized by the drag coefficient of the single model. All platoon members benefit from platooning by experiencing a reduction in drag. The drag reduction is about $15 \%$ for the first model and $60 \%$ for the last one. The drag experienced by the interior platoon members is even lower, with almost 70 and 80 per cent reduction for the second and third models, respectively.

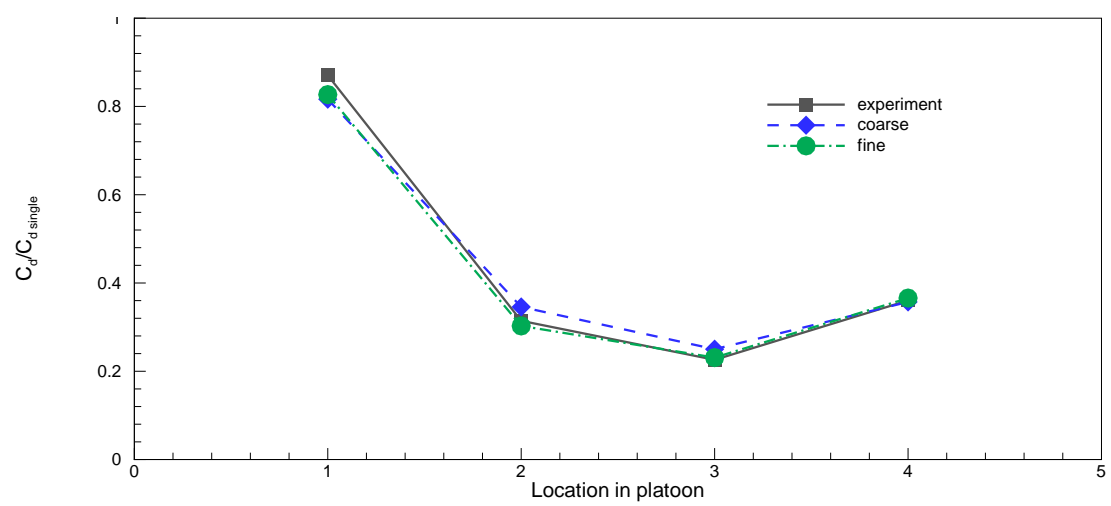

Figure 4: Drag reduction for a four-model platoon.

Figure 4 also illustrates the better behavior of the numerical solution ob- 
tained on the fine grid compared to the numerical solution obtained on the coarse grid. Nevertheless, the results obtained on the coarse grid are already in good agreement with the experimental data.

Figure 5(a) represents the surface pressure coefficient $C_{p}=2\left(p-p_{\infty}\right) / \rho U_{\infty}^{2}$, where $p-p_{\infty}$ is the relative pressure, on the four models. Clearly, the area of high pressure occurring on the front side of the models decreases when the position of the model increases in the platoon. The consequence is a decrease in the drag coefficient when the position of the model is higher. However, it is shown that the effect of the negative pressure occurring on the rear side of the models is higher for the fourth model than for the other models. This is because the fourth model did not have a downstream neighbor. This higher effect of the negative pressure on the rear side of the fourth model explains the higher value of the drag coefficient for this model compared to the second or the third model, figure 4.

Figures 5(b) and 5(c) show the evolution of the pressure coefficient on the 


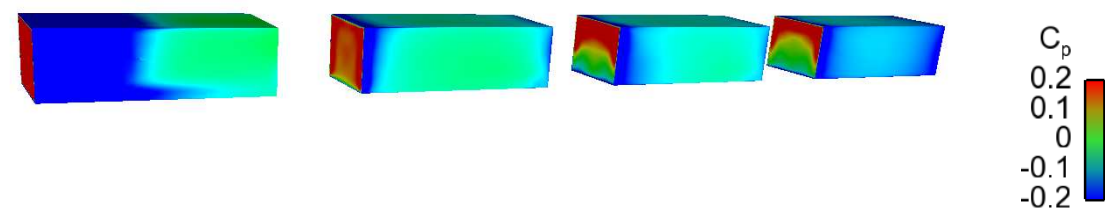

(a) Surface $C_{p}$ on the four platoon members

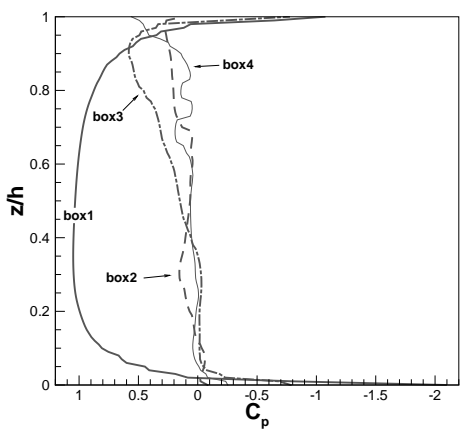

(b) $C_{p}$ on the front side

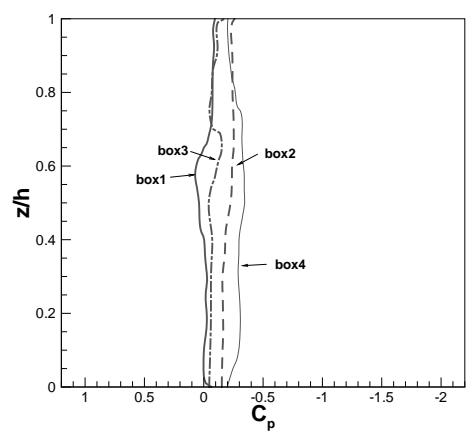

(c) $C_{p}$ on the back side

Figure 5: (a): Pressure coefficients on the four platoon members. (b)-(c): evolution of $C_{p}$ along the middle line of the front and the back side, from the bottom to the top of boxes.

middle line (in the direction of the body width) of the front side and the back side, respectively, for the four models. It is clear that the first model experiences the highest pressure on the front. Compared to the second and the fourth models, the third model shows an increase of the pressure coefficient from the middle of its height. This increase is moved up for the fourth model. For the back side, figure 5(c), it can be seen that the pressure of the first model is almost positive which contributes to reduce the drag coefficient 
and explains the gain of $15 \%$ cited above. The pressure coefficient of the fourth model is clearly the lowest which accounts for the higher value of drag coefficient as compared to the second and the third models.

\subsubsection{Non-uniform Intra-platoon spacing}

Figure 6 shows the evolution of the drag coefficient of the four platoon members when varying the position of the third model. In the experimental work [14], nine different positions were considered, as said previously, $\delta=0, \pm 0.1875, \pm 0.375, \pm 0.5625$ and \pm 0.75 . Here, only five positions were considered: $\delta=0, \pm 0.375$ and \pm 0.75 . Furthermore, a new computational grid was made for each position in order to keep the same resolution. Indeed, it was possible to deform the mesh done for the study of the uniform intra-platoon spacing. However, this deformation, leading to compressed and stretched cells, had unavoidable spurious effects on the results. Therefore, it was definitely more accurate to keep the same resolution in the intra-spacing for all the positions studied by making new computational grids.. The numerical results are compared to the experimental data in figure 6 . In this figure, the results are presented in the platoon order: the first model at the 
top until the fourth model at the bottom. Root Mean Squares (rms) of the drag coefficient signals are also represented in figure 6 on the right $y$-axis to highlight the flow stability.

It can be seen that there is good agreement of the global trends of the LES results with the experimental data. The underestimation of the LES simulations for the first model, 6(a), is still present and explains the discrepancy with the experimental data. The agreement is very good for the third and the fourth models. For these models, drag evolutions are almost straight lines. For the fourth model, 6(d), it shows that the drag coefficient decreases when the third model is getting closer. On the other hand, the results obtained on the second model, 6(b), are poorer than the results of the other models, especially at the position -0.375 and 0.75 . For this second model, the drag behavior is more complex. It shows that there is a dramatic change when the third model moves between the positions 0 and 0.75. Tsuei and Savaş [14] suggested that highly unstable flow exists in the gap between the second and the third model. This unstable flow can explain the more substantial 
difference committed by the LES simulations.

Numerically, the unstable flow occurring on the second model can be highlighted by the value of the rms of the drag coefficient. The rms values of the first model are almost constant and are about $0.02,6(\mathrm{a})$, which means that the flow is relatively stable, i.e. the flow does not show severe hysteresis. These values are higher and more inconstant for the fourth model, 6(d). Nevertheless, they are relatively low, except for the positions $\delta=-0.375$ and $\delta=0.75$. In the same way, the values of the third model are generally upper bounded by 0.03 . The position $\delta=0.375$, is an exception with a value of $0.059,6(\mathrm{c})$. This position coincides with the highest difference in the LES simulations, as compared to the experimental data, on the third model. For the second model, rms values higher than 0.05 are obtained at three different positions: $\delta=-0.375, \delta=0.375$ and $\delta=0.75$, showing the unstable behavior of the flow, 6(b). These three positions correspond to the three most substantial differences in the LES simulations as compared to the experimental data. 
Streamlines of the time-averaged velocity projected onto the central symmetry plane, $y=0$, are shown in figure 7 . These streamlines are plotted for the five positions cited previously: $\delta=0, \pm 0.375$ and \pm 0.75 .

At the position $\delta=0.75,7(\mathrm{a})$, when the third model is the furthest from the fourth model, the near-wake separation bubble of the oscillating model shows two recirculation regions. These recirculation regions do not extend over the gap between the third model and the fourth model, and a part of the airstream impinges the front of the fourth model and induces high pressure there. The high pressure contributes to an increase in the drag coefficient. When the third model is getting closer to the fourth one, the bottom recirculation region is shifted upwards, $7(\mathrm{~b})$, to reach the top of the model at the position $\delta=0,7(\mathrm{c})$. Afterwards, a second recirculation region is created which is attached at the fourth model leading edge, $7(\mathrm{~d})$. Note that, from the position $\delta=0$, the recirculation regions at the rear of the third model are clearly reattached on the sides of the fourth model contributing to reduce the drag of the fourth model. 
When the third model is the furthest from the second model, at the position $\delta=-0.75,7(\mathrm{e})$, the near-wake separation bubble of the second model shows a single recirculation region. This recirculation region is similar to that of the third model at the position $\delta=0.375,7(\mathrm{~b})$. A similarity can be seen between the near-wake separation bubble of the second model at the position $\delta=0.375,7(\mathrm{~b})$, and the near-wake separation bubble of the third model at the position $\delta=-0.375,7(\mathrm{~d})$. This time, the distance between the second and the third models and between the third and the fourth models is the same. At the position $\delta=0,7(\mathrm{c})$, the near-wake separation bubble behind the second model shows a large lower recirculation region. At the position $\delta=-0.375,7(\mathrm{~d})$, the lower recirculation region is replaced by an upper recirculation region.

The recirculation regions behind the second model are clearly shorter at position $\delta=0.375,7(\mathrm{~b})$, as compared to the original position $\delta=0,7(\mathrm{c})$. It means this size is constrained by the third model and, then, the velocity in- 
side these recirculation regions is higher. Consecutively, the pressure is lower and the drag coefficient of the second model is higher, at position $\delta=0.375$, than at position $\delta=0$, see 6 (b). At position $\delta=0.75$, it seems that the high pressure occurring on the front side of the second model acts on the back side of the second model, see 7(a), and, then, decrease the drag coefficient of the second model, see 6(b).

Table 4 summarizes, for the five positions studied, the global drag coefficient, of the vehicle-platoon defined as:

$$
C_{d \text { global }}=\frac{\sum_{i=1}^{4} C_{d i}}{4 C_{d \text { single }}}
$$

where $C_{d i}$ represents the drag coefficient of the $i-t h$ platoon member. By comparison with the uniform intra-platoon spacing, $\delta=0$, the position $\delta=$ -0.75 yields a $5 \%$ decrease in the global drag. On the other hand, the position $\delta=0.375$ involves an increase of $13 \%$. These results suggest that a non-uniform intra-platoon spacing can be more efficient if the positions of the models are right. However, the choice of the locations of the models is not trivial. 


\begin{tabular}{l|l|l|l|l|l}
\hline$\delta$ & -0.75 & -0.375 & 0 & 0.375 & 0.75 \\
\hline$C_{\text {dglobal }}$ & 0.418 & 0.457 & 0.442 & 0.501 & 0.456 \\
\hline
\end{tabular}

Table 4: Global drag of the vehicle platoon for the different positions of the oscillating model. 


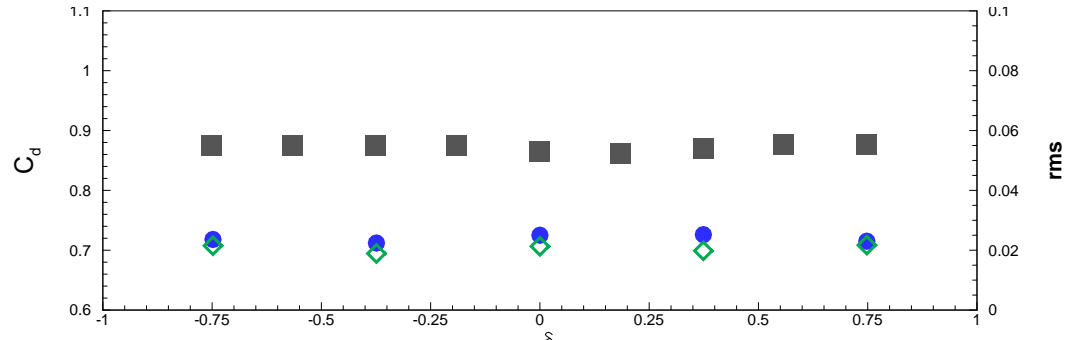

(a) box1

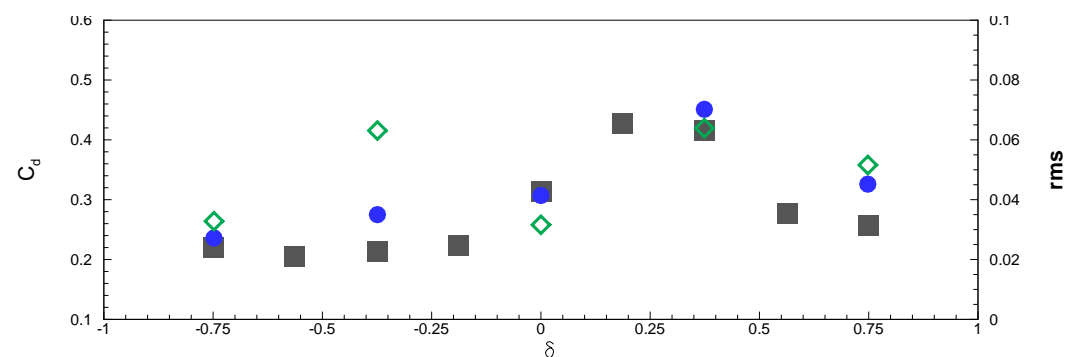

(b) box2

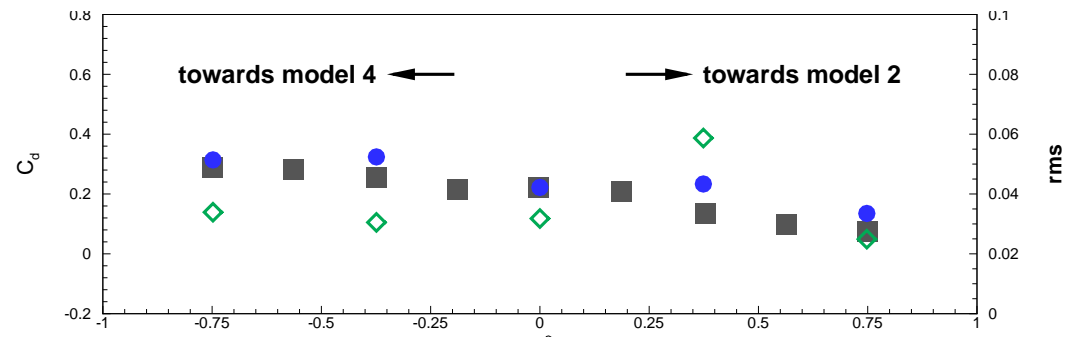

(c) box3

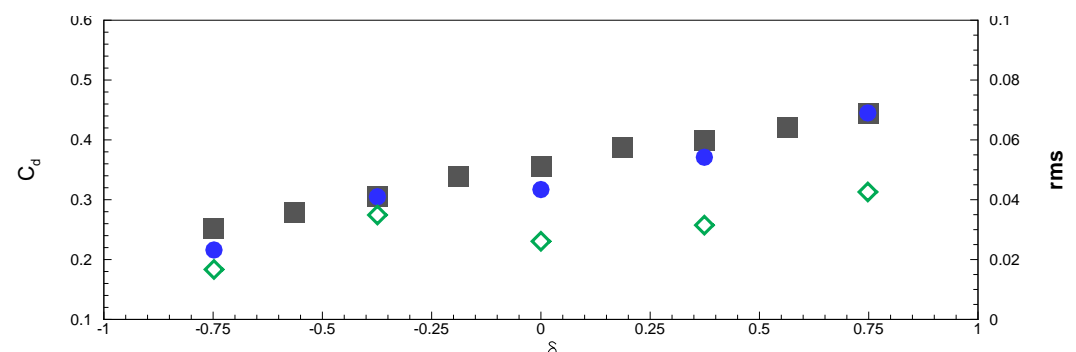

(d) box4

Figure 6: Comparisons of the drag coefficients of the four models for the five different positions of the third model $\delta=0, \pm 0.375, \pm 0.75$ : ( $)$ experimental data, (•) LES results. Root Mean Square (rms) of the drag coefficient $(\diamond)$, on the right axis. 


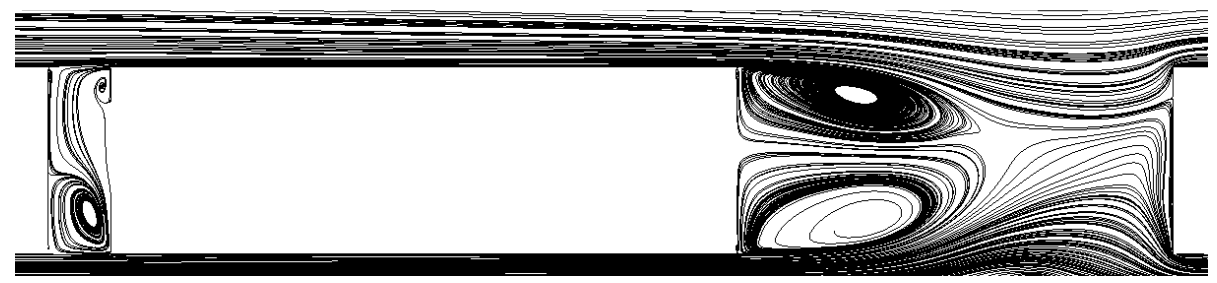

(a) $\delta=0.75$

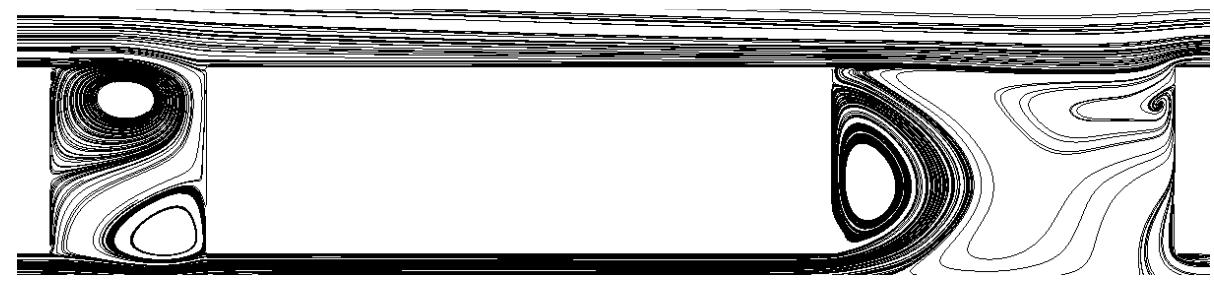

(b) $\delta=0.375$

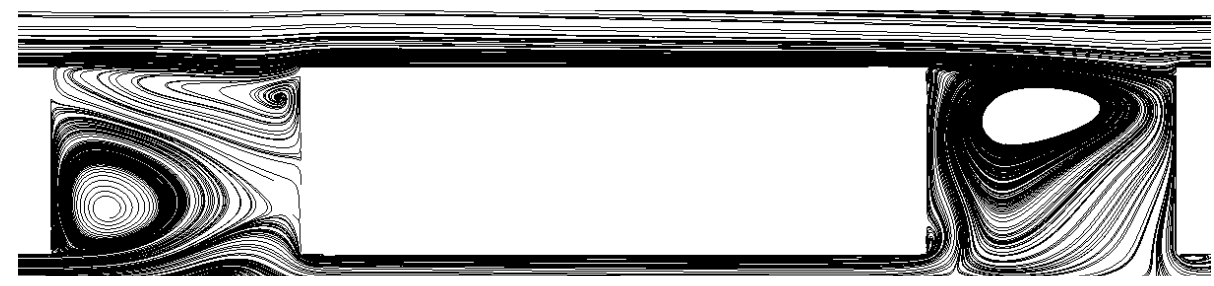

(c) $\delta=0$

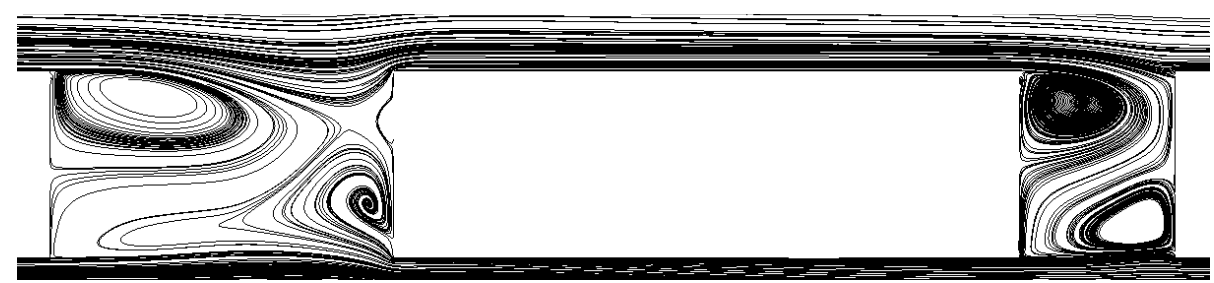

(d) $\delta=-0.375$

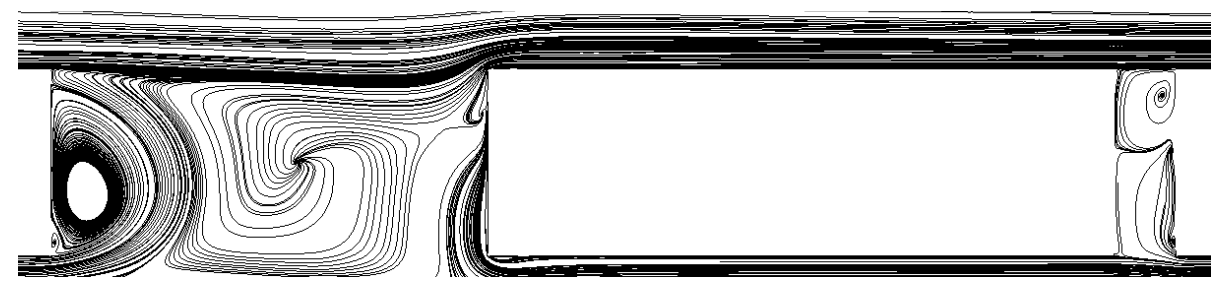

(e) $\delta=-0.75$

Figure 7: Streamlines of the time-averaged velocity projected on plane $y=0$. The flow direction is from the left to the right. 


\subsection{Results of the dynamic simulations}

\subsubsection{Case1: $\delta_{a m p}=0.375$ and $f=2 \mathrm{~Hz}$}

Figure 8 shows the experimental data and the numerical results of the drag coefficient experienced by the three static models, i.e. the first, the second and the fourth. These coefficients are plotted over one complete period of oscillation. The amplitude was $\delta_{a m p}=0.375$ and the frequency was $f=2 \mathrm{~Hz}$. The last graph of this figure, $8(\mathrm{~d})$, represents the position of the oscillating model, giving a better comprehension of the drag fluctuations. The difference between the maximum and the minimum values on each curve is labelled $\Delta C_{d}$. This amplitude quantifies the effect of the oscillating model acting on the other members of the platoon. This case is referred as to Case1 in the following text.

The agreement of the results of the dynamic model with the experimental data is good. It is shown that the trends of the curves are well reproduced. Nevertheless, the maximum value of the drag coefficient of the second model, 8(b), is overestimated, while the minimum value of the drag coefficient of the 
fourth model, 8(c), is underestimated. These two behaviors yield an overestimation of the drag coefficient amplitude, $\Delta C_{d}$, for both the second and the fourth models. The fourth model experiences a periodic drag evolution, i.e. the drag coefficient of the fourth model is a linear function of the position of the oscillating model. Clearly, the drag decreases when the third model gets closer and increases when the third model gets further away. Moreover, effects of the third model on the drag amplitude of the fourth model are clearly smaller than effects of the third model on the drag amplitude of the second model. Actually, the total drag is dominated by the pressure drag caused by flow separation at the rear end of the vehicles. It is understood that the effects of the oscillating model are more substantial on its upstream neighbor than on its downstream neighbor. Moreover, for the second model, there is no periodic behavior. Finally, it can be noted that effects of the oscillating model on the first model are almost non-existent because the disturbed flow is blocked by the second model. 


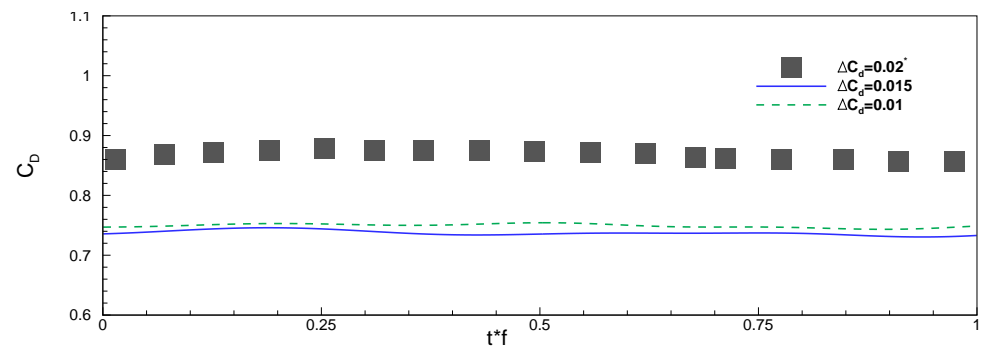

(a) box1

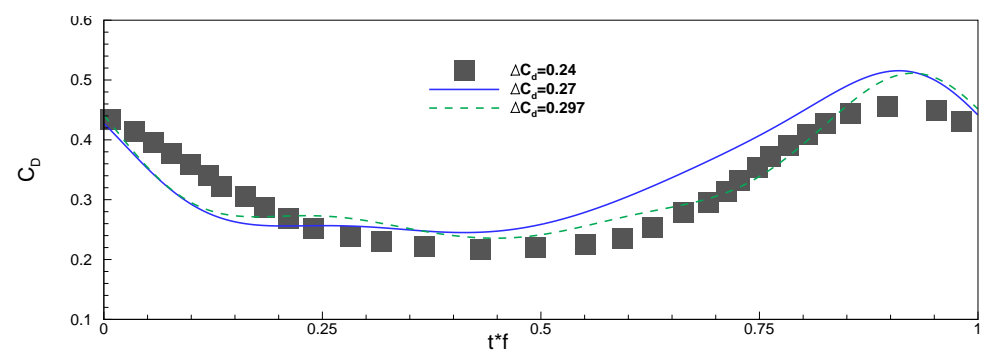

(b) box2

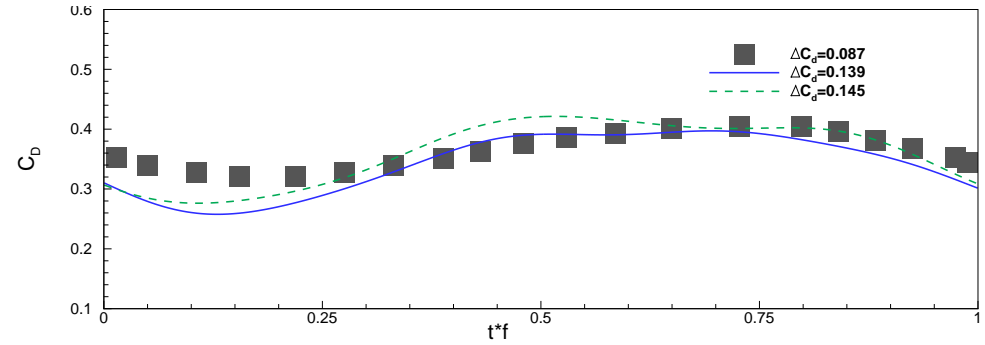

(c) box4

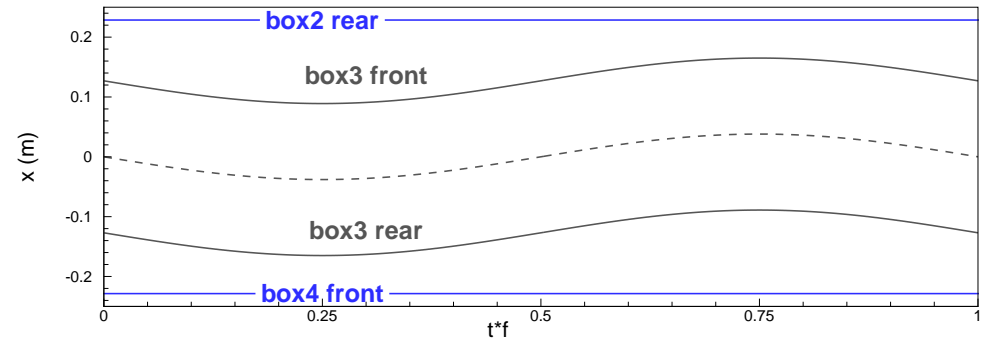

(d) position of box3

Figure 8: Effect of dynamic oscillation $\left(\delta_{a m p}=0.375\right.$ and $f=2 \mathrm{~Hz}$ ). (a)-(c) drag coefficient plotted over one period of oscillation: $(\boldsymbol{\square})$ experimental, $(-)$ coarse mesh, $(-\rightarrow$ fine mesh. (d) position of the oscillating model. 
It is interesting to look why the drag coefficient of the second model is almost inverted, i.e. the drag coefficient is minimum when the oscillating model is distant, while it is maximum for a close oscillating model. It is expected that the oscillating model leads to an increase of the base pressure of the second model when it is approaching. This will in turn lead to a decrease of the drag of the second model. Figure 9 shows the velocity vectors projected onto the symmetry plane $\mathrm{y}=0$ for two positions of the oscillating model: close to the fourth model 9 (a) and close to the second one 9(b). It can be seen that the oscillating model reduces the region of flow with recirculation when it approaches the second model, see 9(b), which contributes to increase the velocity. The pressure, then, decreases with a value $C_{p}<-0.3$ at the top of the second model back side, see $9(\mathrm{~d})$. When the oscillating model is close to the fourth one, figure $9(\mathrm{a})$, the recirculation regions are not so compressed and the pressure is higher: $C_{p}>-0.1$ for almost the total height of the model. These observations confirm the remarks already done in section 3.1.2. 


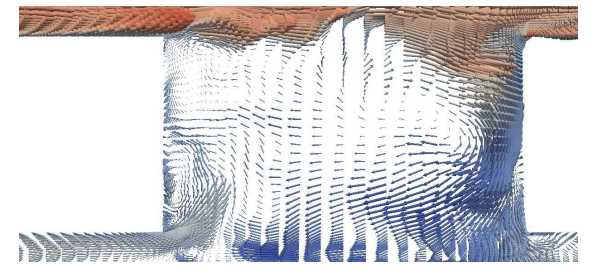

(a) $t \times f=0.25$

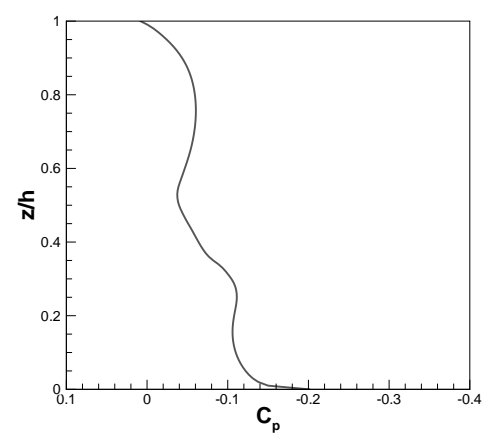

(c) $C_{p}$ on back side, $t \times f=0.25$

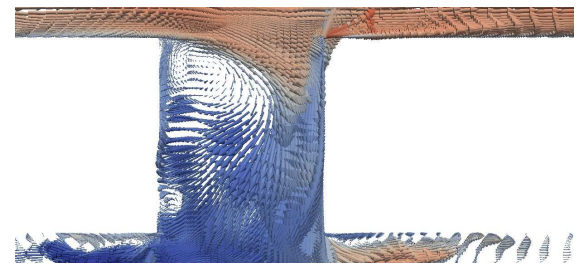

(b) $t \times f=0.75$

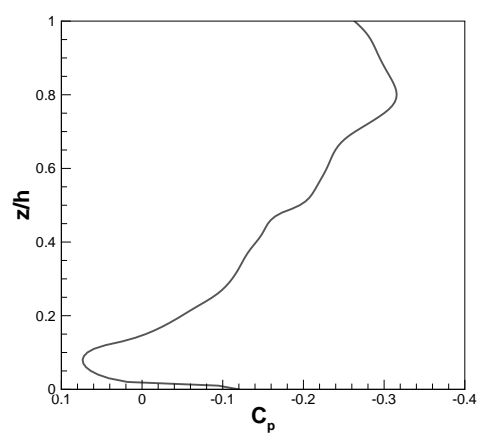

(d) $C_{p}$ on the back side, $t \times f=0.75$

Figure 9: (a)-(b): Velocity vector, behind the second model, colored by its first component value onto the middle plane $\mathrm{y}=0$. For both figures, second model is on the left. (c)-(d) Pressure coefficient on the middle line of the second model back side

\subsubsection{Case2: $\delta_{a m p}=0.75$ and $f=1 \mathrm{~Hz}$}

Figure 10 shows the experimental data and the numerical results of the drag coefficient experienced by the three static models, i.e. the first, the second and the fourth. These coefficients are plotted over one complete period of oscillation. The last graph of this figure, 10(d), represents the position of the oscillating model giving a better understanding of the drag fluctuations. 
The amplitude in this case was $\delta_{a m p}=0.375$ and the frequency $f=1 \mathrm{~Hz}$. This case is referred as to Case 2 in the following text.

It can be seen that the drag coefficient of the first model, see 10(a), is still slightly affected by the oscillation of the third model. The results of the dynamic model on the fourth model are in good agreement with the experimental data, 10(c). The drag coefficient of the fourth model remains periodic and, thus, easily predictable. On the other hand, the results of the second model differ from the experimental data. The minimum value is well predicted: between $t \times f \simeq 0.15$ and $t \times f \simeq 0.5$, see figure $10(\mathrm{~b})$. This minimum value occurs when the oscillating model is far from the second model. At $t \times f=0.5$, the oscillating model returned to its original position. The first increasing part, between $t \times f \simeq 0.5$ and $t \times f \simeq 0.625$, is also well reproduced. But the dramatic change around $t \times f \simeq 0.625$ is strongly overestimated by the dynamic model results. Actually, at this position, the experimental drag coefficient experiences a slight decrease yielding a local minimum. This local minimum occurs when the oscillating vehicle is about at its closest position 
from the second model. When the oscillating model keeps away from the second model, the drag increases once again, at $t \times f \simeq 0.9$. This increasing part is also well reproduced by the numerical simulations. Unfortunately, the numerical curves are shifted due to the overestimation of the previous decrease.

The displacement amplitude of 0.75 pushes the current numerical simulation to its limit. Indeed, such an amplitude involves that cells located in the deformation zones are scaled by $1 / 8$ th. This can have a substantial effect on the quality of the calculation. Moreover, the gap between the second and the third models is critical in a physical phenomena point of view, as was already shown by the quasi-steady results. The combination of these two elements can explain the poor behavior of the numerical results on the second model.

However, the numerical decrease of the second model drag coefficient around $t \times f=0.75$ can be explained by the observations done in section 3.1 .2 on the streamlines behind the second model. Indeed, here $t \times f=0.75$ 
corresponds to the position $\delta=0.75$, and it was concluded that the high pressure occurring on the front side of the third model acts on the back side of the second model, then contributing to decrease the drag coefficient. 


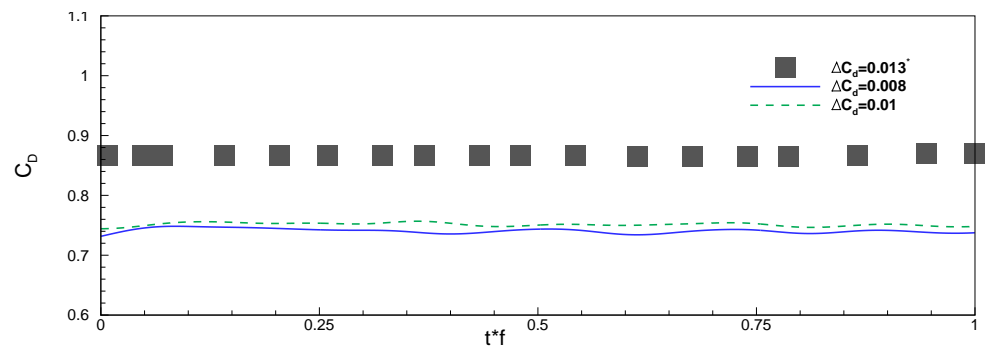

(a) box1

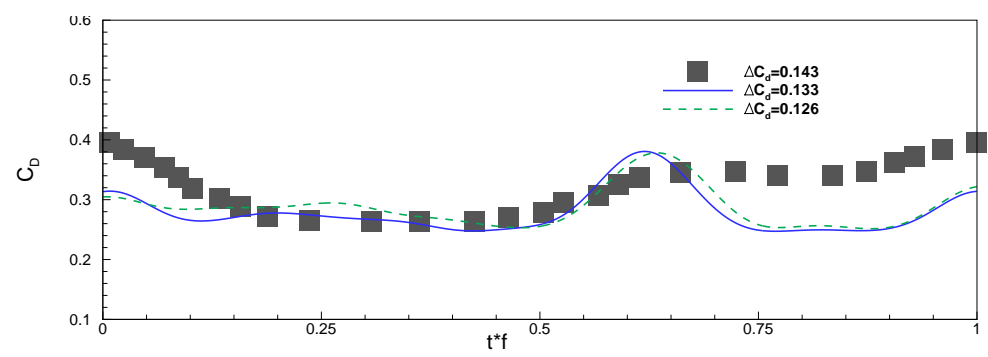

(b) box2

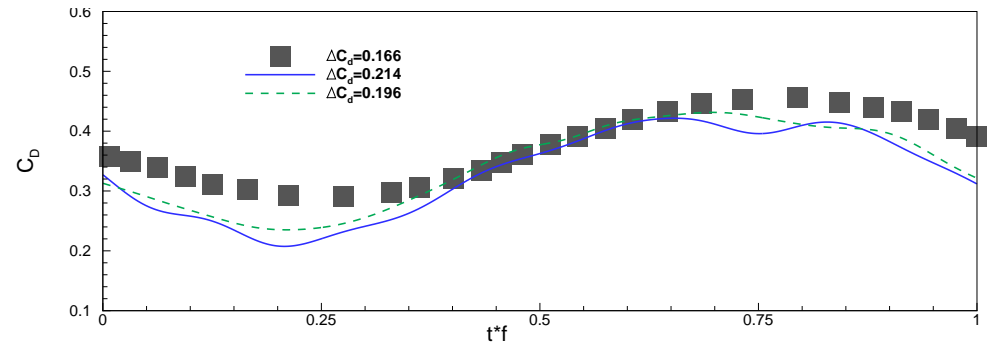

(c) box4

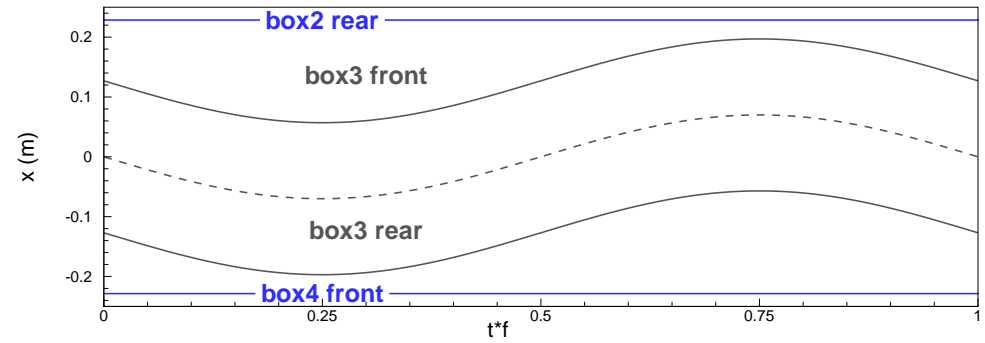

(d) position of box3

Figure 10: Effect of dynamic oscillation $\left(\delta_{a m p}=0.75\right.$ and $\left.f=1 \mathrm{~Hz}\right)$. (a)-(c) drag coefficient plotted over one period of oscillation: $(\mathbf{\square})$ experimental, $(-)$ coarse mesh, $(-\rightarrow$ fine mesh. (d) position of the oscillating model. 


\subsection{Hysteresis effects}

Figure 11 shows the comparison between the quasi-steady results presented in section 3.1 and the dynamic results. The dynamic results consist of the Case $1 \delta_{a m p}=0.375$ and $f=2 \mathrm{~Hz}$ of section 3.2.1, the Case $2 \delta_{a m p}=0.75$ and $f=1 \mathrm{~Hz}$ of section 3.2 .2 and, in addition, the Case $3 \delta_{a m p}=0.75$ and $f=2 \mathrm{~Hz}$. With this new dynamic case, studies of the frequency effect as well as the displacement amplitude effect are possible. The drag coefficients illustrated in figure 11 are presented as functions of the position of the oscillating model $\delta$.

Once again, effects on the first model are weak, and the steady values are close to the unsteady results, 11(a). Drag coefficients of the other models show hysteresis effects in their behavior in the two parts of the sweep between the extreme positions $-\delta_{a m p}$ and $\delta_{a m p}$. This behavior results in different values of drag coefficients for the same position of the oscillating model. The third model (i.e. the oscillating model) and the fourth model show equivalent hysteresis effects in an amplitude point of view. Indeed, for the third model, 
the maximum amplitude, i.e. the maximum difference on a same curve for the same abscissa, is $\Delta C_{d} \simeq 0.11$ at position $\delta=-0.375$ for both Case2 and Case3, 11(c). This amplitude is even smaller for Case1. Moreover, the frequency seems to have no effect on the drag coefficient of the oscillating model. For the fourth model, the maximum amplitude is also $\Delta C_{d} \simeq 0.11$ for Case1 and Case3, 11(d), i.e. for the two cases with the same frequency. This time, the hysteresis effects are weaker for Case2, with the smallest frequency.

The results obtained on the second model are the most interesting, 11(b). Indeed, they show different behaviors highlighting the unpredictable flow occurring in the gap between the second and the third models. For Case1, the maximum amplitude is $\Delta C_{d} \simeq 0.22$, which is twice the maximum amplitude of the third and the fourth models. The curve shows a drop-like shape. Actually, this kind of shape can already be seen on the curves of the third and the fourth models. It translates the fact that, for instance, when the oscillating model is close to the fourth model, the effect on the drag coefficient of the second model is weak, while, when the oscillating model is close to the 
second model, the second model experiences a significant drag change. The drag evolution is strongly different for the two highest amplitudes, Case2 and Case3. For these cases, the drag curves are affected by the overestimation of the chaotic change discussed previously.

Figure 12 shows the surface pressure coefficient on the models and an isosurface of relative pressure at position $\delta=0$ for three situations. These situations are: 12(a) when the third model is moving towards the second model, 12(b) when the third model is steady and 12(c) when the third model is moving towards the fourth model. The position $\delta=0$ was chosen because it shows large $\Delta C_{d}$ for all the dynamic results. However, differences in flows between the quasi-steady and the dynamic flow conditions were found for all positions. It is clearly shown that the dynamic simulations, $12(\mathrm{a})$ and $12(\mathrm{c})$, involve large differences of the flow structures compared to the quasi-steady simulation 12(b). These differences affect the surface pressure on the models and, thereby, the drag coefficient. When the third model is moving towards the second model, 12(a), the flow structures between the third and the fourth 
models are clearly larger than the flow structures in the other two situations. This explains the higher value of drag coefficient for the fourth model when the third model is moving towards the second one, 11(d). Similarly, when the third model is going towards the fourth model, 12(c), the flow structures between the second and the third models are larger than the flow structures in the two other situations. The consequence is a higher value of drag coefficient for the second model. 


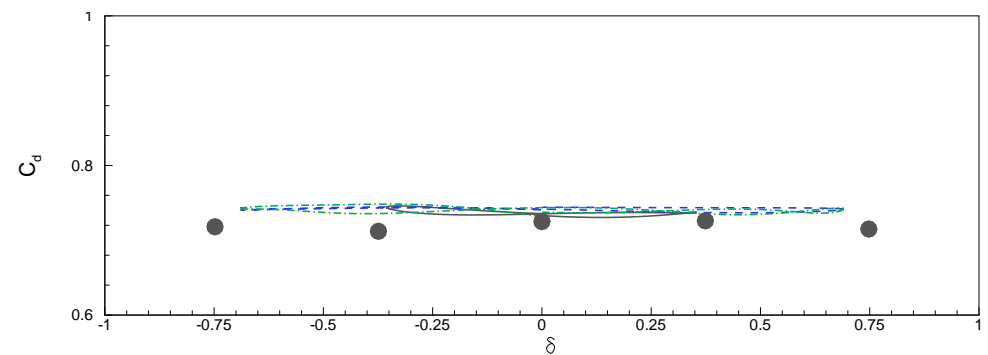

(a) box1

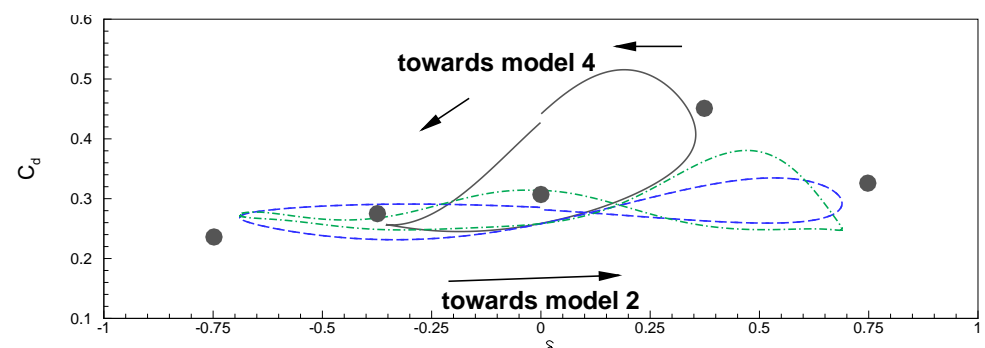

(b) box2

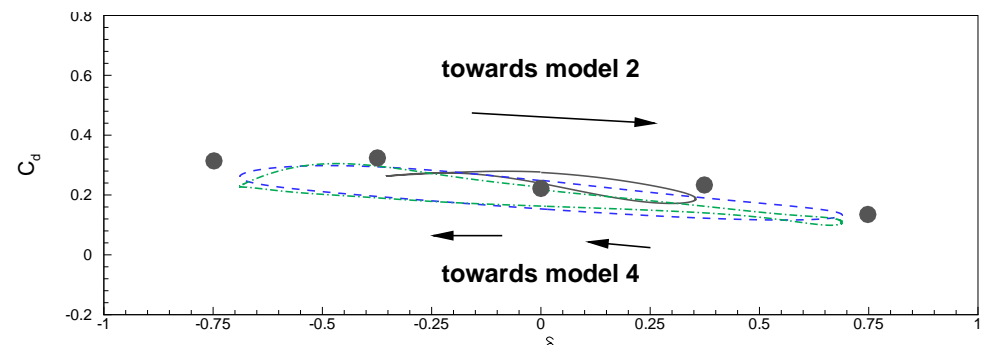

(c) box3

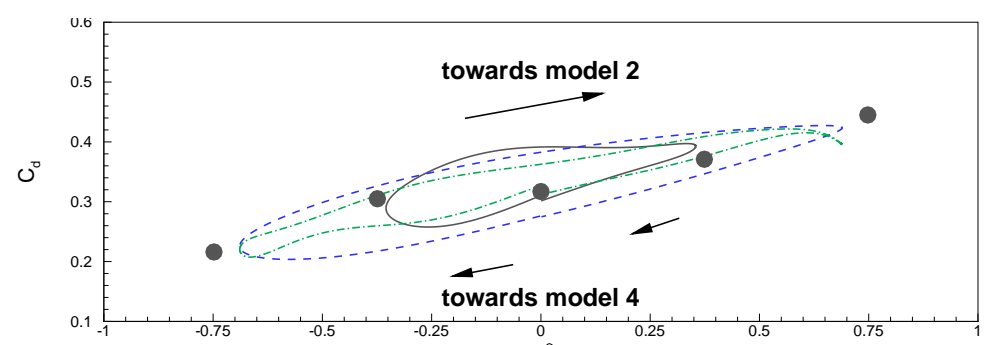

(d) box4

Figure 11: Comparisons of quasi-steady and dynamic results. $(\bullet)$ steady, $(---) \delta_{a m p}=0.75$ and $f=1 \mathrm{~Hz},(-) \delta_{a m p}=0.375$ and $f=2 \mathrm{~Hz},(--) \delta_{a m p}=0.75$ and $f=2 \mathrm{~Hz}$. 


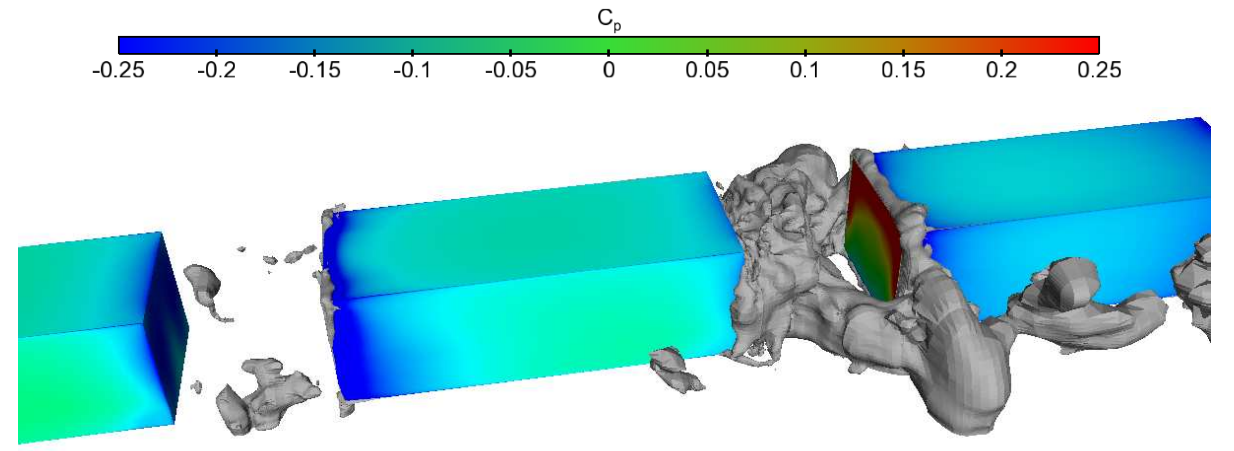

(a) $\delta=0$, motion towards model 2

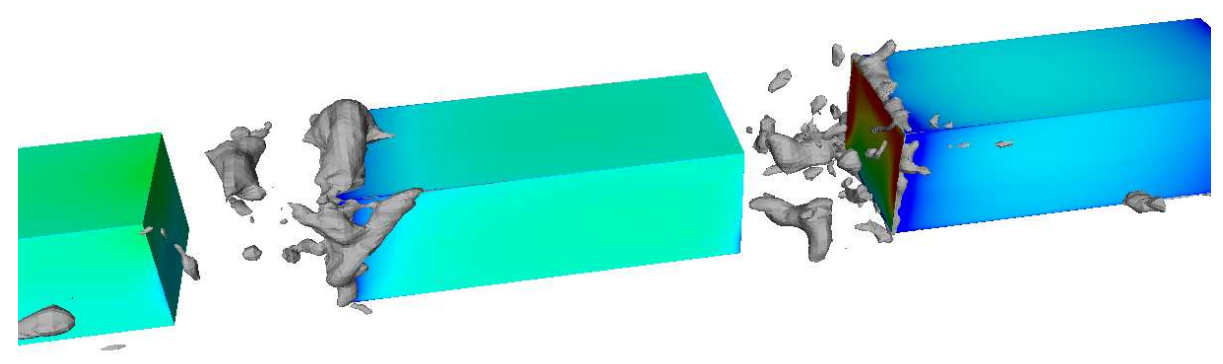

(b) $\delta=0$, quasi-steady

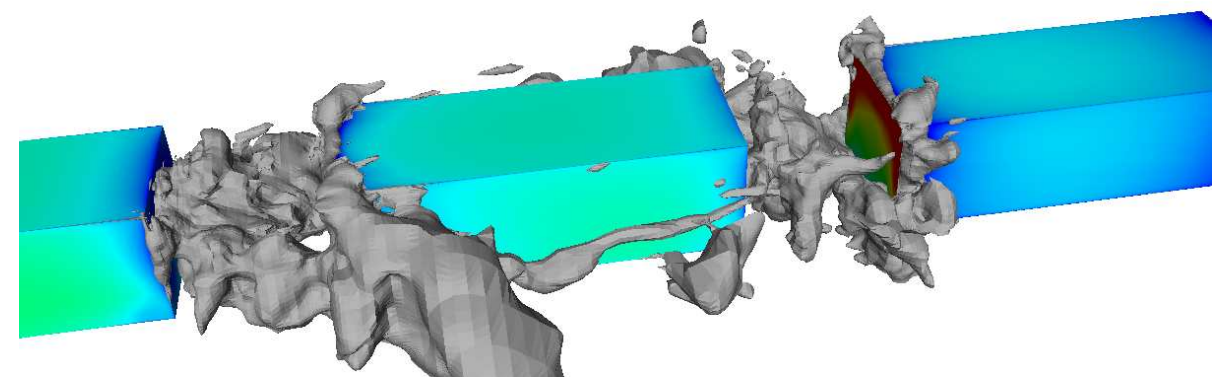

(c) $\delta=0$, motion towards model 4

Figure 12: One isosurface of relative pressure, $p=-50 \mathrm{~Pa}$. The bodies are colored with surface pressure coefficient $C_{p}$. 
For Case1, the drag coefficient of the second model shows a large hysteresis effect. To improve the understanding of this phenomenon, figure 13 shows the velocity vector and the pressure coefficient behind the second model at position $\delta=0$, when the oscillating model moves towards the fourth one 13(a), and towards the second one 13(b). In addition to figures 9 (a) and 9(b), figures 13(a) 13(b) complete a total period of oscillation of the third model. The drag coefficient of the second model is strongly higher when the oscillating model moves towards the fourth one with a difference $\Delta C_{d} \simeq 0.2$ at position $\delta=0$, i.e. about $65 \%$ of the drag coefficient obtained in the steady case, see section 3.1. It is confirmed by the lower pressure behind the second model in figure 13(a). In section 3.2.1, it was shown that the approach of the oscillating model decreases the base pressure of the second model and, then, increases the drag coefficient. This pressure decrease, involved by the acceleration of the flow, continues even when the oscillating model is going further to reach a maximum of drag coefficient around the position $\delta=0.25$. 


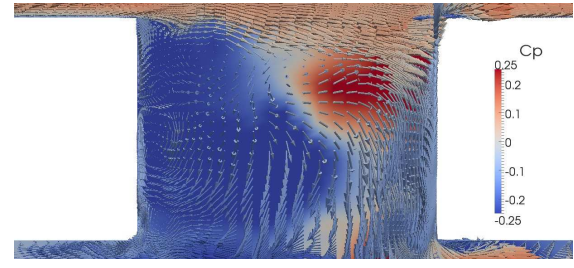

(a) $\delta=0$, towards model 4

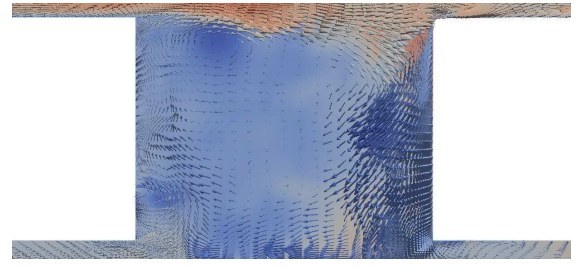

(b) $\delta=0$, towards model 2

Figure 13: Velocity vector, behind the second model, colored by its first component value onto the middle plane $\mathrm{y}=0$ and pressure coefficient contours. The second model is on the left.

\section{Conclusions}

The LES of flow around a four-vehicle platoon was performed to explore the effects involved by the oscillation of one platoon member. It was confirmed that all platoon members benefit from platooning by experiencing drag reduction. Dynamic model clearly showed that the longitudinal oscillations of a platoon member have strong influences on the drag force of the neighbor members. The upstream neighbor especially experienced a chaotically changing drag, and this behaviour has been explained. Other vehicles, i.e. the vehicles that are not neighbors, do not seem to be affected. LES proved to be able to accurately predict change in the aerodynamic drag force caused by the oscillation of a platoon member. 
The most important limitation of the present numerical methodology is in an accurate prediction of the flow on the upstream neighbor when the oscillation amplitude is large. For this type of amplitude, another grid strategy, such as an adaptive grid or overlapping grids, should be explored. Nevertheless, the initial purpose was to study effects of oscillations naturally occurring in a platoon, which are obviously smaller than the maximum amplitude $\delta_{a m p}=0.75$ studied in this paper.

Longitudinal oscillation does not constitute the only transient phenomenon which has an undeniable effect on the platoon aerodynamic performance. In the same way, the lateral position is difficult to maintain, and lateral oscillation influences aerodynamic performances. Such lateral oscillations, or perhaps a combination of longitudinal and lateral oscillations, should be considered in future work. 


\section{Acknowledgments}

This work is supported financially by the Safer, Vehicle and Traffic Safety

Centre at Chalmers. Software licenses were provided by AVL List GMBH.

Computations were performed at SNIC (Swedish National Infrastructure for Computing) at the Center for Scientific Computing at Chalmers (C3SE) and the National Supercomputer Center (NSC) at Liu.

\section{References}

[1] Abdel Azim, A. F., Abdel Gawad, A. F., 2000. A flow visualization study of the aerodynamic interference between passenger cars. SAE 2000-010355 .

[2] Koening, K., Roshko, A., 1985. An experimental study of geometrical effects on the drag and flow field of two bluff bodies separated by a gap. Journal of Fluids Mechanics 156, 167-204.

[3] Krajnović, S., 2009. LES of flows around ground vehicles and other bluff bodies. Philosopical Transactions of the Royal Society A 367, 1899, $2917-2930$.

[4] Krajnović, S., Bjerklund, E., Basara B., 2009. Simulations of the flow around high-speed train meeting each other at the exit of a tunnel. 21st International Symposium of Dynamics of Vehicles on roads and Tracks. 
[5] Krajnović, S., Bengtsson, A., Basara, B., 2011. Large eddy simulation investigation of the hysteresis effects in the flow around an oscillating ground vehicle. ASME: Journal of Fluids Engineering 133, 121103.

[6] Marcu, B., Browand, F., 1999. Aerodynamic forces experienced by a 3-vehicle platoon in crosswind. SAE 1999-01-1324.

[7] Martinuzzi, R. J., Havel, B., 2004. Vortex shedding from two surfacemounted cubes in tandem. International Journal of Heat and Fluid Flow $25(3), 364-372$.

[8] Osth, J., Krajnović, S., 2012. The flow around a simplified tractor-trailer model studied by large eddy simulation. Journal of Wind Engineering and Industrial Aerodynamics 102, 36-47.

[9] Pagliarella, R. M., Watkins, S., Tempia, A., 2007. Aerodynamic Performance of Vehicles in Platoons: The Influence of Backlight Angles. SAE 2007-01-1547.

[10] Paik, J., Sotiropoulos, F., Porté-Agel, F., 2009. Detached eddy simulation of flow around two wall-mounted cubes in tandem. International Journal of Heat and Fluid Flow 30 (2), 286-305.

[11] Schito, P., Braghin, F., 2012. Numerical and experimental investigation on vehicles in platoon. SAE Int. J. Commer. Veh. 2012-01-0175.

[12] Smagorinsky, J., 1963. General circulation experiments with the primirtive equations.Monthly Weather Review 91 (3), 99-164. 
[13] Tsuei, L., Savaş, O., 2000. A wind tunnel investigation of the transient aerodynamic effects on a four-car platoon during passing maneuvers. SAE 2000-01-0875.

[14] Tsuei, L., Savaş, O., 2001. Transient aerodynamics of vehicle platoons during in-line oscillations. Journal of Wind Engineering and Industrial Aerodynamics 89, 1085-1111.

[15] Watkins, S., Vino, G., 2008. The effect of vehicle spacing on the aerodynamics of a representative car shape. Journal of Wind Engineering and Industrial Aerodynamics 96, 1232-1239.

[16] Zabat, M., Frascaroli, S., Browand, F., 1994. Drag measurements on 2, 3 and 4 car platoons. SAE 940421.

[17] Zdravkovich, M., 1977. Review of flow interference between two circular cylinders in various arrangements. Trans. ASME, J. Fluids Eng. 99, 618-633. 